\title{
O papel da videolaparoscopia para o tratamento de úlceras pépticas perfuradas: revisão de literatura
}

\section{The role of videolaparoscopy for the treatment of perforated peptic ulcers: review article}

\author{
David Sales Rocha Pintoํ. Francisco de Assis Costa². \\ 1 Residente de Cirurgia Vascular, Hospital Geral de Fortaleza (HGF), Fortaleza, Ceará, Brasil. 2 Chefe do Serviço de Cirurgia \\ Geral, Hospital Geral de Fortaleza (HGF), Fortaleza, Ceará, Brasil.
}

\section{RESUMO}

A doença ulcerosa péptica possui prevalência estimada em 5\% a 15\% da população. A perfuração é uma complicação da doença ulcerosa péptica, consistindo em uma emergência cirúrgica que possui taxa de mortalidade de $15 \%$. O tratamento dessa complicação tem evoluído para abordagens menos invasivas visando a melhores resultados e estadias hospitalares. O presente estudo tem por objetivo apresentar o papel da abordagem laparoscópica no tratamento de úlceras pépticas perfuradas.

Palavras-chave: Úlcera péptica. Laparoscopia. Úlcera péptica perfurada.

\section{ABSTRACT}

The peptic ulcer disease has an estimated prevalence of $5 \%$ to $15 \%$ of the population. The perforation is a complication of peptic ulcer disease, consisting of a surgical emergency which has a $15 \%$ mortality rate. Its therapy has evolved to less invasive approaches aiming to better outcomes and hospital stays. This study aims to present the role of the laparoscopic approach in the treatment of perforated peptic ulcers.

Keywords: Peptic ulcer. Laparoscopy. Peptic ulcer perforation.

Autor correspondente: David Sales Rocha Pinto, Rua Paula Ney, 100, Aldeota, Fortaleza, Ceará. CEP: 60.140-200. Telefone: +55 85 99785-2109. E-mail: davidsrpinto@gmail.com

Conflito de interesses: Não há qualquer conflito de interesses por parte de qualquer um dos autores.

Recebido em: 31 Ago 2017; Revisado em: 17 Mai 2018; Aceito em: 17 Mai 2018. 


\section{INTRODUÇÃO}

A doença ulcerosa péptica é uma doença caracterizada pela perda de continuidade da mucosa do estômago ou do duodeno. $\mathrm{O}$ desequilíbrio entre fatores de proteção versus fatores de agressão à mucosa está implicado na gênese desta entidade patológica.

As causas mais comuns de úlcera péptica incluem a infecção por Helicobacter pylori (48\%) e o uso de anti-inflamatórios não esteroidais (5 a 20\%). Outras causas incluem o uso de medicações (como bifosfonatos e agentes quimioterápicos), malignidade (Câncer de estômago, linfoma gástrico), estados de hipersecreção gástrica (como na Síndrome de Zollinger-Ellison) e estresse agudo (politraumatizados, por exemplo).

Possui prevalência estimada em 5 a $15 \%$ da população, com incidência de $10 \%$ ao longo da vida. Ocorre em $70 \%$ das vezes, entre os 25 e 64 anos de idade. Devido ao maior conhecimento da patogênese da doença ulcerosa péptica e no avanço do tratamento clínico desta doença, tem se observado declínio na incidência, na necessidade de hospitalização e no tratamento cirúrgico eletivo desta patologia. Porém, o percentual de pacientes que necessitam de cirurgia de emergência não foi alterado, aproximadamente 7\% dos hospitalizados. $^{2}$

A perfuração é uma complicação da doença ulcerosa péptica. Ela consiste na perda de continuidade de todas as camadas da parede do órgão envolvido. Seu tratamento consiste em uma emergência cirúrgica, devido à alta taxa de mortalidade $(15 \%){ }^{2}$

A perfuração ocorre em 2 a $10 \%$ das úlceras pépticas. Comumente envolve a parede anterior do duodeno (60\%), embora possa se manifestar no antro gástrico (29\%) e na curvatura menor do estômago $(20 \%) .^{3}$

O diagnóstico de uma úlcera péptica perfurada é baseado na história clínica, no exame físico e na rotina radiológica de abdome agudo.

Histórico de sintomas prévios para úlcera péptica pode ajudar a direcionar as hipóteses diagnósticas, apesar de 40 a $50 \%$ dos pacientes terem como manifestação clínica inicial a perfuração (principalmente em usuários de anti-inflamatórios não esteroidais e em idosos). ${ }^{4}$

Úlceras perfuradas manifestam-se por dor epigástrica súbita e intensa.

O exame físico clássico de um paciente com úlcera perfurada revela defesa abdominal involuntária difusa (abdome em tábua), com descompressão brusca dolorosa difusa, principalmente em epigastro (peritonite).

A rotina radiológica de abdome agudo é uma ferramenta eficaz para o diagnóstico. Pode-se encontrar ar livre entre a cúpula diafragmática direita e o fígado no raio-X de tórax em ortostase (Figura 1). Outros exames de imagem, como a Tomografia Computadorizada de Abdome, podem ser úteis em casos de histórias atípicas.

Figura 1. Ar livre entre fígado e cúpula diafragmática e entre estômago e cúpula diafragmática (setas brancas).

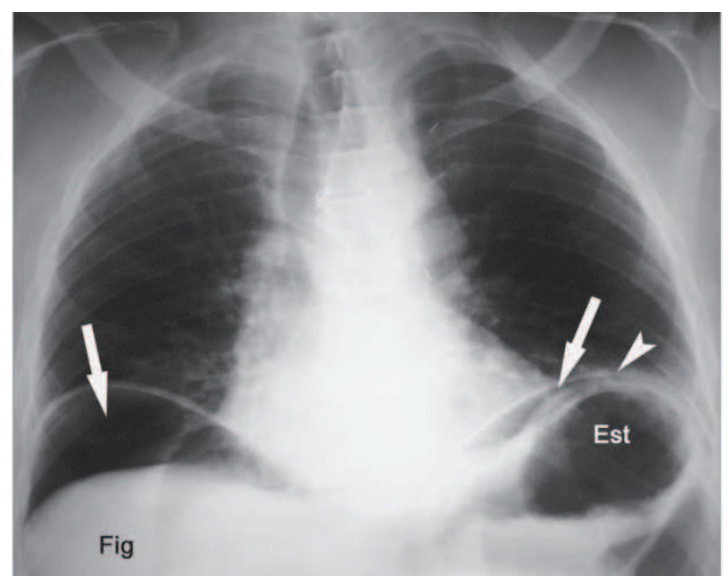

Fonte: Lopes AC, Reibscheid S, Szejnfeld J. Abdome agudo. Clínica e imagem. São Paulo: Atheneu; 2004.

Nas últimas décadas, tem se dado importância às abordagens minimamente invasivas. Estudos científicos mostram que menores incisões cirúrgicas estão associadas a menor estresse metabólico ao trauma, culminando em menor morbimortalidade e menor estadia hospitalar.

A laparoscopia está ganhando mais espaço na cirurgia abdominal de emergência, com expansão constante do seu campo de aplicação. As principais vantagens desta técnica incluem a facilidade no diagnóstico e a menor taxa de complicações em relação à abordagem cirúrgica aberta. A necessidade de recursos tecnológicos e de equipe treinada incluem limitações para a prática desta técnica.

Diversos estudos realizados em centros experientes têm evidenciado a eficácia da abordagem laparoscópica para as úlceras pépticas perfuradas, o que levantou a dúvida sobre qual técnica cirúrgica é mais eficaz, se correção laparoscópica ou cirurgia convencional.

\section{OBJETIVOS}

O presente estudo tem por objetivo analisar a importância da laparoscopia no tratamento da úlcera péptica perfurada. Resultados pós-operatórios e custo-eficiência, bem como a comparação com a técnica cirúrgica convencional foram contemplados neste trabalho.

\section{METODOLOGIA}

Foi realizada pesquisa bibliográfica em literatura pertinente, nacional e internacional, abrangendo artigos de revisão, 
artigos originais, consensos e meta-análises da base de dados Medline. Foram utilizados os descritores Peptic Ulcer; Laparoscopy; Peptic Ulcer Perforation conforme o DeCS.

Os artigos selecionados para a revisão tiveram como critérios: relevância e publicação em revistas conhecidas e confiáveis. Foram descartados, após leitura, os artigos que não apresentavam metodologia adequada ou não abordavam a área de interesse.

\section{REVISÃO DE LITERATURA}

\section{Introdução}

Roscoe Reid Graham realizou com sucesso patch omental pela primeira vez em 1929 para um paciente com insulinoma. ${ }^{5}$ Mouret desenvolveu o primeiro reparo laparoscópico em 1989, utilizando patch omental e cola de fibrina. ${ }^{6}$ Desde então, a factibilidade do reparo laparoscópico tem sido validado por diversos estudos.

A cirurgia aberta é o tratamento clássico para as úlceras pépticas perfuradas. Com relação às úlceras duodenais, o procedimento consiste em sutura primária com ou sem reforço de retalho de omento vascularizado. A técnica pode ser por via aberta (Figura 2) ou por via laparoscópica (Figura 3). As perfurações acima de 3 centímetros podem ser difíceis de fechar, fazendo-se necessário a aposição de retalho saudável (utiliza-se omento ou serosa jejunal), duodenostomia (drenagem do orifício perfurado com sonda Foley) ou até antrectomia com reconstrução à Billroth II. ${ }^{7}$

Figura 2. Reparo aberto.
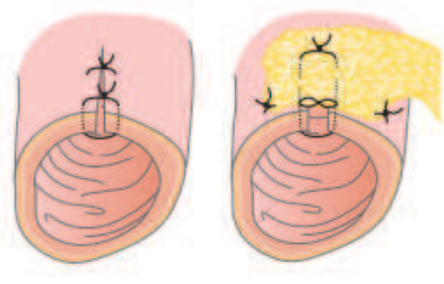

a Pimary cibsure
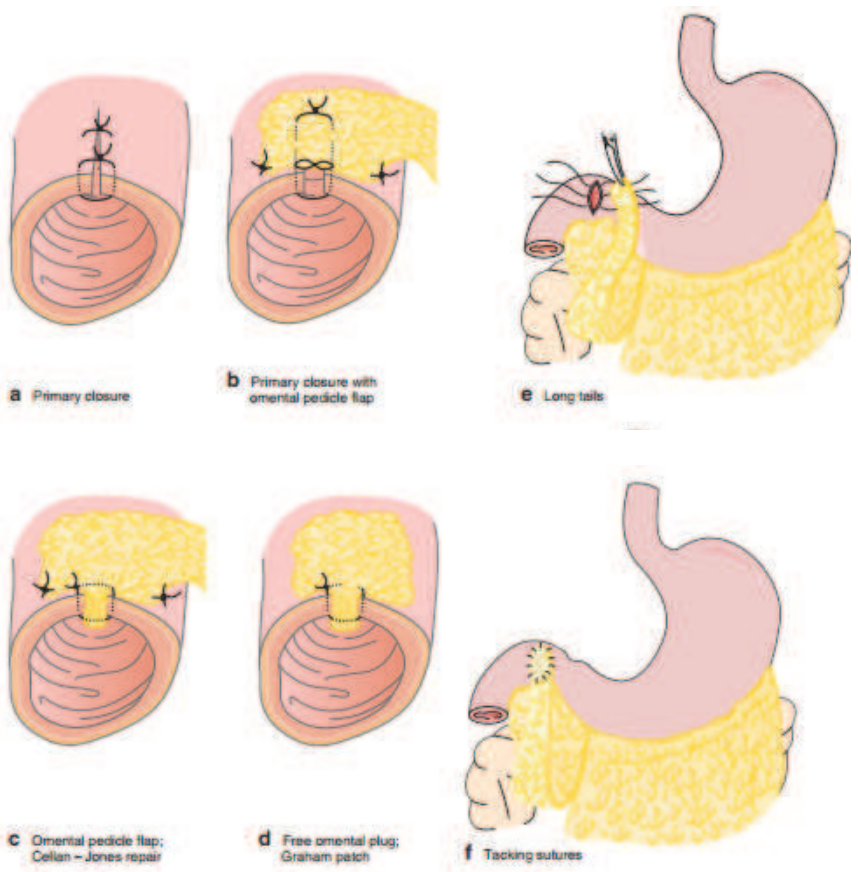

Fonte: Søreide K, Thorsen K, Søreid JA. Strategies to improve the outcome of emergency surgery for perforated peptic ulcer. Br J Surg. 2014 Jan;101(1):e51-64.
Figura 3. Reparo laparoscópico.

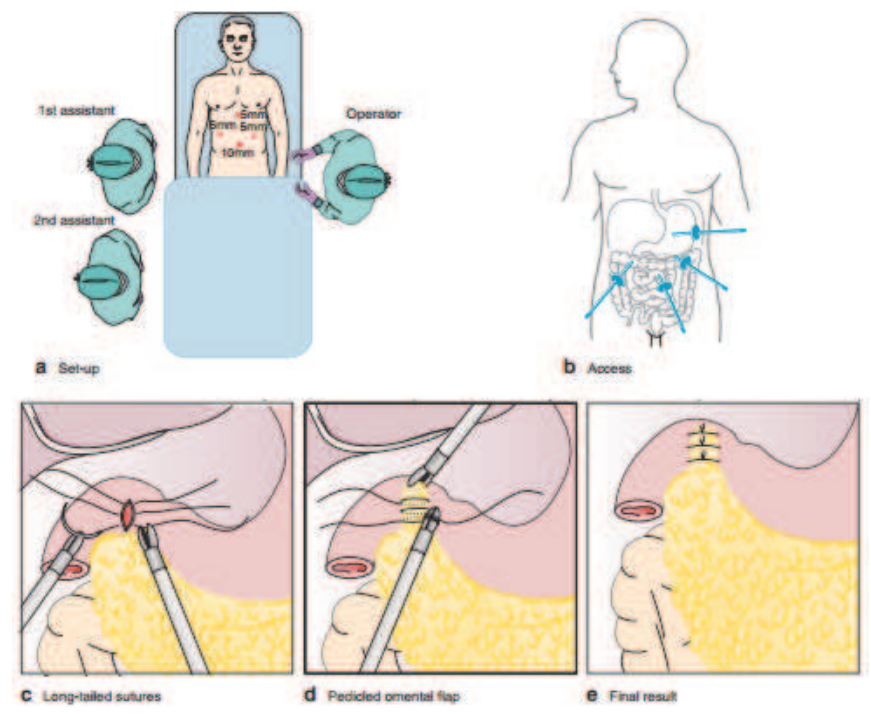

Fonte: Søreide K, Thorsen K, Søreid JA. Strategies to improve the outcome of emergency surgery for perforated peptic ulcer. Br J Surg. 2014 Jan;101(1):e51-64.

As úlceras gástricas tipo I em pacientes estáveis exigem gastrectomia distal com anastomose à Billroth I. Nos pacientes instáveis com úlcera gástrica tipo I, fechamento com retalho omental é a conduta ideal. As úlceras gástricas tipos II e III se comportam como úlceras duodenais, tendo a realização de sutura com patch omental, com ou sem vagotomia troncular, o procedimento de escolha. ${ }^{7}$

\section{Tipos de reparo laparoscópico}

O tratamento laparoscópico padrão consiste em lavagem da cavidade abdominal com sutura primária da perfuração reforçada com a adição de patch omental ao procedimento. Outra forma de abordar envolve a realização de sutura sem o patch omental.

Turner et al. relataram que a sutura sem um remendo omental resultaria em uma taxa de mortalidade significativamente mais elevada do que com um remendo. No entanto, uma das explicações pode ser que a maioria dos casos na sua série foram úlceras gástricas perfuradas em vez de perfuração duodenal. ${ }^{8}$

Lucivicius et al. avaliou 13 estudos prospectivos e 12 estudos retrospectivos e concluiu que o método de reparação deve melhor ser julgado pelas propriedades da borda da úlcera. ${ }^{9}$

Ates e Dirican et al. concluíram que o simples reparo sutura de PPU sem remendo omental é boa opção para o reparo com base em critérios que incluem a duração dos sintomas, o Mannheim Peritoneal Index (MPI), o escore da ASA (American Society of Anesthesiologists) e o tamanho da perfuração (>12mm). ${ }^{10}$

Um estudo retrospectivo realizado em 2013 envolvendo 179 pacientes operados de junho de 2005 a dezembro de 2012, mostrou que a sutura simples possuía tempos de permanência hospitalar, tempo para retorno da ingestão oral, complicações 
pós-operatórias e resultados cirúrgicos semelhantes à abordagem laparoscópica com sutura reforçada com patch omental, com a vantagem de apresentar tempo cirúrgico significativamente menor. ${ }^{11}$

No entanto, o reparo laparoscópico envolvendo sutura com reforço omental permanece ainda como o procedimento padrão.

\section{Reparo laparoscópico versus cirurgia aberta}

Em 2013, foi publicada uma meta-análise realizada por um grupo alemão. A pesquisa teve como proposta comparar os resultados da laparoscopia versus a cirurgia aberta, utilizando ensaios clínicos disponíveis na literatura. ${ }^{12}$

Foram selecionados somente ensaios clínicos randomizados. De 290 estudos coletados a partir de uma revisão nas bases de dados Medline, Cochrane e Excepta Medica, 4 ensaios clínicos randomizados (Quadro 1) foram selecionados, totalizando 289 pacientes. (Tabela 1)

O objetivo era comparar o reparo laparoscópico versus a cirurgia aberta em relação às seguintes variáveis: mortalidade, tempo de cirurgia, complicações maiores e necessidade de reoperação.

O conjunto de dados disponíveis demonstrou resultados homogêneos para as variáveis de desfecho de mortalidade, complicações e taxa de reoperação (Tabela 2). Não foi possível identificar significância estatística para qualquer uma dessas variáveis, embora o odds ratio seja consistentemente a favor do reparo laparoscópico, o que sugere um potencial erro estatístico tipo II. A abordagem laparoscópica resultou numa menor taxa de complicações menores (10\% versus $23 \%)$.

Quadro 1. Características dos Ensaios Clínicos Randomizados.

\begin{tabular}{|c|c|c|c|c|c|}
\hline Autor & $\begin{array}{c}\text { Ano de } \\
\text { publicação }\end{array}$ & $\begin{array}{l}\text { Quantidade } \\
\text { de centros } \\
\text { participantes }\end{array}$ & Período do tratamento & Critérios de inclusão & Critérios de exclusão \\
\hline Lau et al & 1996 & 1 & $\begin{array}{l}\text { Agosto de } 1992 \text { a setembro } \\
\text { de } 1994\end{array}$ & $\begin{array}{l}\text { Diagnóstico clínico de } \\
\text { úlcera péptica perfurada }\end{array}$ & $\begin{array}{l}\text { 1. Úlcera complicada } \\
\text { 2. Úlcera sangrante } \\
\text { 3. Cirurgia abdominal prévia } \\
\text { 4. Doença cardiopulmonar } \\
\text { grave }\end{array}$ \\
\hline Lau et al & 1998 & 1 & $\begin{array}{l}\text { Setembro de } 1995 \text { a julho de } \\
1996\end{array}$ & Idade entre 17 e 69 anos & $\begin{array}{l}\text { 1. Úlcera sangrante } \\
\text { 2. Imunossupressão }\end{array}$ \\
\hline Siu et al & 2002 & 1 & $\begin{array}{l}\text { Janeiro de } 1994 \text { a junho de } \\
1997\end{array}$ & $\begin{array}{l}\text { 1. Clinical diagnosis of PPU } \\
\text { 2. Age }>16 \text { years }\end{array}$ & $\begin{array}{l}\text { 1. Bleeding ulcer } \\
\text { 2. Cirurgia abdominal prévia } \\
\text { 3. Obstrução pilórica }\end{array}$ \\
\hline Bertleff et al & 2009 & 9 & $\begin{array}{l}\text { Março de } 1999 \text { a julho de } \\
2005\end{array}$ & $\begin{array}{l}\text { Diagnóstico clínico de } \\
\text { úlcera péptica perfurada }\end{array}$ & $\begin{array}{l}\text { 1. Cirurgia abdominal prévia } \\
\text { 2. Gravidez }\end{array}$ \\
\hline
\end{tabular}

Tabela 1. Dados demográficos e cirúrgicos das populações estudadas.

\begin{tabular}{|c|c|c|c|c|c|c|c|c|}
\hline Autor & $\begin{array}{l}\text { Número de } \\
\text { pacientes } \\
\text { (Lap/Ab) }\end{array}$ & Age (Lap/Ab) & $\begin{array}{l}\text { Razão } \\
\text { homem/ } \\
\text { mulher }\end{array}$ & $\begin{array}{l}\text { Escore ASA } \\
\text { (Lap/Ab) }\end{array}$ & $\begin{array}{l}\text { Escore Boey } \\
\text { (Lap/Ab) }\end{array}$ & $\begin{array}{l}\text { Tamanho da } \\
\text { perfuração }(\mathrm{mm}) \\
\text { (Lap/Open) }\end{array}$ & $\begin{array}{l}\text { Patch } \\
\text { omental }\end{array}$ & $\begin{array}{l}\text { Taxa de } \\
\text { conversão }\end{array}$ \\
\hline Lau et al & $45(24 / 21)$ & $52(52 / 53)$ & $4.6: 1$ & NR & $\begin{array}{l}0.27 \\
(0.29 / 0.24)\end{array}$ & $\mathrm{NR}(6 \mathrm{~mm} / 5 \mathrm{~mm})$ & Sim & $25 \%(6 / 24)$ \\
\hline Lau et al & $22(12 / 10)$ & NR & NR & NR & NR & NR & Sim & $25 \%(3 / 12)$ \\
\hline Siu et al & $121(63 / 58)$ & $55(54 / 56)$ & $4.3: 1$ & $\begin{array}{l}1.72 \\
(1.7 / 1.7)\end{array}$ & $\begin{array}{l}0.28 \\
(0.24 / 0.33)\end{array}$ & $5.0(5.2 / 4.7)$ & Sim & $14 \%(9 / 63)$ \\
\hline Bertleff et al & $101(52 / 49)$ & NR $(66 / 59)^{*}$ & $1.5: 1$ & $\begin{array}{l}\text { NR } \\
(1.0 / 1.5)^{*}\end{array}$ & NR & NR $(10.0 / 7.0)^{*}$ & $\begin{array}{l}\text { Casos } \\
\text { misturados }\end{array}$ & $8 \%(4 / 52)$ \\
\hline
\end{tabular}

NR: não relatado; ASA: American Society of Anesthesiologists; lap: laparoscópica; ab: aberta.

Dados numéricos são relatados em valores médios.

*valores médios. 
Tabela 2. Resultados.

\begin{tabular}{|c|c|c|c|c|c|c|c|c|}
\hline Autor & $\begin{array}{c}\text { Tempo } \\
\text { Cirúrgico } \\
\text { (Min) } \\
\text { (Lap/Ab) }\end{array}$ & $\begin{array}{c}\text { VAS }^{ \pm} \\
(\mathbf{L a p} / \mathbf{A b})\end{array}$ & $\begin{array}{l}\text { Tempo (em dias) } \\
\text { para tolerar } \\
\text { dieta oral } \\
\text { (Lap/Ab) }\end{array}$ & $\begin{array}{c}\text { Complicações } \\
\text { menores } \\
\text { (Lap/Ab), } \mathbf{n} \\
(\%)\end{array}$ & $\begin{array}{c}\text { Complicações } \\
\text { maiores } \\
\text { (Lap/Ab), } \mathbf{n} \\
(\%)\end{array}$ & $\begin{array}{c}\text { Reoperação } \\
\text { (Lap/Ab), n } \\
(\%)\end{array}$ & $\begin{array}{c}\text { Estadia } \\
\text { hospitalar } \\
\text { (dias) } \\
\text { (Lap/Ab) }\end{array}$ & $\begin{array}{c}\text { Mortalidade } \\
\text { (Lap/Ab), n } \\
(\%)\end{array}$ \\
\hline Lau et al & $87(113 / 57)$ & NR $(4 / 5)^{*}$ & NR $(4 / 4)^{*}$ & $2 / 4(8 / 19)$ & $2 / 1(8 / 5)$ & $0 / 1(0 / 5)$ & $\operatorname{NR}(5 / 5)^{*}$ & $0 / 1(0 / 5)$ \\
\hline Lau et al & NR $(96 / 35)^{*}$ & NR & NR & NR & NR & NR & NR & NR \\
\hline Siu et al & $47(42 / 52)$ & $4.9(3.5 / 6.4)$ & $\mathrm{NR}(4 / 5)^{*}$ & $7 / 14(11 / 24)$ & $3 / 8(5 / 14)$ & $5 / 1(8 / 5)$ & $\operatorname{NR}(6 / 7)^{*}$ & $1 / 3(2 / 5)$ \\
\hline Bertleff et al & $\mathrm{NR}(75 / 50)^{*}$ & $(3.8 / 5.2)^{*}$ & NR & NR & NR & NR & $\operatorname{NR}(6.5 / 8)^{*}$ & $2 / 4(4 / 8)$ \\
\hline
\end{tabular}

n: número absoluto; NR: não relatado; lap: laparoscópica; ab: aberta; *Valores médios; $† 24$ horas depois da cirurgia.

Dados numéricos são relatados como valores médios

Várias limitações para o estudo devem ser levadas em conta. A meta-análise identificou somente 4 ensaios clínicos randomizados. Três dos estudos eram de qualidade metodológica adequada, enquanto um dos estudos não forneceu dados suficientes para uma avaliação estatística. A população do estudo cumulativo foi baixa e a força da análise foi, portanto, limitada.

\section{Risco de conversão cirúrgica}

Em 2015, um estudo coreano retrospectivo analisou dados de 77 pacientes submetidos a reparo laparoscópico simples (sem patch omental) para úlceras perfuradas durante o período de janeiro de 2007 a setembro de $2013 .^{13}$

Dos 77 pacientes submetidos ao procedimento, 8 necessitaram de conversão para cirurgia aberta. Os pacientes foram divididos em 2 grupos, cirurgia totalmente laparoscópica e os que necessitaram de conversão cirúrgica.

As características dos pacientes, os achados intra-operatórios, as complicações pós-operatórias, as taxas de conversão e as taxas de vazamento pela sutura foram investigados.

O único fator de risco identificado na análise multivariável do estudo foi o tamanho da úlcera. Úlceras com tamanho acima de 9 milímetros foram associadas a maior taxa de conversão cirúrgica.

Úlceras acima de 9 milímetros e duração da perfuração acima de 12,5 horas foram associadas a maior taxa de vazamento pela sutura.

A conversão cirúrgica, no entanto, não está associada a maior morbidade e mortalidade pós-operatória do que em relação aos pacientes submetidos inicialmente a laparotomia. ${ }^{14,15}$

\section{Custo-eficiência}

Foi observado menor estadia hospitalar, menor taxa de mortalidade e menor custo hospitalar no grupo de pacientes submetidos a laparoscopia, porém, sem diferença estatisticamente significante.

Um estudo realizado em 2014 teve como objetivo comparar o custo-eficiência da abordagem laparoscópica versus a cirurgia convencional. Os dados foram retirados do Nationwide Inpatient Sample entre os períodos de 2007 a 2010.

Os pacientes que se submeteram à operação para úlcera péptica perfurada foram divididos com base na abordagem laparoscópica ou aberta. As variáveis analisadas foram tempo de internação, mortalidade e custos totais.

Um total de 5.361 pacientes foi identificado: 5.219 no grupo aberto e 142 no grupo laparoscópico. Os pacientes do grupo laparoscópico eram mais jovens (50,5 versus 60,0 anos, $p$ $<0,001)$ e tiveram uma incidência menor na apresentação de sepse $(8,5$ versus $14,8 \%, p=0,034)$ e choque $(2,1$ versus $7,7 \%, \mathrm{p}=0,012)$.

$\mathrm{Na}$ análise univariada, o grupo da laparoscopia teve menor tempo de internação ( 7,0 versus 8,0 dias, $\mathrm{p}<0,001)$, taxas menores de mortalidade (3,5 versus $8,1 \%, \mathrm{p}=0,048)$, e receberam alta com maior frequência (79,6 versus $68,1 \%$, $\mathrm{p}=0,025)$. A média de encargos totais foi menor no grupo laparoscópico (44.095 versus 52.055 dólares, $\mathrm{p}=0,019$ ). $\mathrm{Na}$ análise multivariada, não houve diferença estatisticamente significante para nenhuma das variáveis do desfecho.

\section{CONCLUSÃO}

Ainda permanece incerto o papel ideal do reparo laparoscópico nas úlceras pépticas perfuradas. A cirurgia aberta se mantém como o padrão-ouro. Apesar da maioria dos estudos se mostrarem favoráveis à técnica laparoscópica, não há resultados estatisticamente significantes que favoreçam uma técnica sobre a outra. A experiência em laparoscopia tem crescido nos diversos centros. Portanto, novos ensaios clínicos randomizados são necessários para tentar responder essa questão ainda não resolvida. 


\section{REFERÊNCIAS}

1. Kurata JH, Nogawa AN. Meta-analysis of risk factors for peptic ulcer. Nonsteroidal antiinflammatory drugs, Helicobacter pylori, and smoking. J Clin Gastroenterol. 1997;24(1):2-17.

2. Sabiston DC Júnior. Tratado de Cirurgia. 18.ed. Rio de Janeiro: Editora Elsevier; 2015.

3. Behrman SW. Management of complicated peptic ulcer disease. Arch Surg. 2005;140(2):201-8.

4. Hilton D, Iman N, Burke GJ, Moore A, O’Mara G, Signorini D, et al. Absence of abdominal pain in older persons with endoscopic ulcers: a prospective study. Am J Gastroenterol. 2001;96(2):380-4.

5. De la Fuente SG, Pappas TN. Roscoe Reid Graham (1890 to 1948): the man of the patch. Curr Surg. 2002;59(4):428-9.

6. Mouret P, Francois Y, Vignal J, Barth X, Lombard-Platet R. Laparoscopic treatment of perforated peptic ulcer. Br J Surg. 1990;77(9):1006.

7. Sabiston DC Júnior, Townsend MC. Tratado de cirurgia. 19. ed. Rio de Janeiro: Elsevier; 2015.

8. Turner WW Junior, Thompson WM Junior, Thal ER. Perforated gastric ulcers. A plea for management by simple closures. Arch Surg. 1988;123(8):960-4.
9. Lunevicius R, Morkevicius M. Management strategies, early results, benefits, and risk factors of laparoscopic repair of perforated peptic ulcer. World J Surg. 2005;29(10):1299-1310.

10. Ates M, Dirican A. The simple suture laparoscopic repair of peptic ulcer perforation without an omental patch. Surg Endosc. 2012;26(1):289.

11. ME Abd Ellatif, AF Salama, AF Elezaby, HF El-Kaffas, A Hassan, A Magdy, et al Laparoscopic repair of perforated peptic ulcer: Patch versus simple closure. Int J Surg. 2013;11(9):948-51.

12. Antoniou SA, Antoniou GA, Koch OO, Pointner R, Granderath FA. Meta-analysis of laparoscopic versus open repair of perforated peptic ulcer. JSLS. 2013;17(1):15-22.

13. Kim JH, Chin HM, Bae YJ, Jun KH. Risk factors associated with conversion of laparoscopic simple closure in perforated duodenal ulcer. Int J Surg. 2015;15:40-4.

14. Zimmermann M, Hoffmann M, Laubert T, Jung C, Bruch HP, Schloericke E. Conversion of laparoscopic surgery for perforated peptic ulcer: a single-center study. Surg Today. 2015;45(11):1421-8.

15. Wright GP, Davis AT, Koehler TJ, Scheeres DE. Cost-efficiency and outcomes in the treatment of perforated peptic ulcer disease: Laparoscopic versus open approach. Surgery. 2014;156(4):1003-7.

\section{Como citar:}

Pinto DS, Costa FA. O papel da videolaparoscopia para o tratamento de úlceras pépticas perfuradas: revisão de literatura. Rev Med UFC. 2018 jul-set;58(3):68-73. 